\title{
Five-Year Data of Clinical Characteristics and Laboratory Findings of Hospitalized Hemophilic Patients in Dr. Hasan Sadikin General Hospital
}

\author{
Dina Marlina, ${ }^{1}$ Lelani Reniarti, ${ }^{2}$ Fifi Veronica ${ }^{3}$ \\ ${ }^{1}$ Faculty of Medicine Universitas Padjadjaran, ${ }^{2}$ Department of Child Health Faculty of Medicine \\ Universitas Padjadjaran/Dr. Hasan Sadikin General Hospital, Bandung, ${ }^{3}$ Department of Anatomy \\ Faculty of Medicine Universitas Padjadjaran
}

\begin{abstract}
Background: Hemophilia A has the highest incidence, more than 80\% of 172.323 cases worldwide in 2012. It is stated that clinical characteristics of hemophilia A is worse than others, so it is required to prove and to know further about the clinical characteristics and severity likelihood in all hemophilic patients in order to prevent re-bleeding and re-injury and also for a better medical response.

Methods: A retrospective cross-sectional study was carried out to 43 medical records of hospitalized hemophilic patients from 2009 to 2013 in Dr Hasan Sadikin General Hospital. The inclusion criteria were a complete patient identity (name, age, sex), written chief complaint, complete physical examination (bleeding, edema, hematoma, hemarthrosis, anemic symptoms) and laboratory test results (factor level, hemoglobin, hematocrit, platelet and Activated Partial Thromboplastin Time). The data was collected from August-October 2014, analyzed and presented using frequency distribution.

Results: Most of the patients were 5-10 years old, male and had hemophilia A. The most common complaint was external bleeding, followed by edema. From 43 patients, $38(88 \%)$ cases were classified as severe factor deficiency, had mild to severe anemia, however the platelet count in most of the cases was in normal value. About 91\% cases had prolonged Activated Partial Thromboplastin Time in moderate to severe level.

Conclusions: Similar with other studies worldwide, most of the hospitalized hemophilic patients have hemophilia A. Most of the patents has moderate to severe bleeding with laboratory test result between moderate to severe level as well. [AMJ.2016;3(4):605-9]
\end{abstract}

Keywords: Bleeding, hemophilia, laboratory findings, severity

\section{Introduction}

Hemophilia is an X-linked disease caused by mutation in sex chromosome resulting in protein deficiency named coagulation factors $^{1,2}$ Among several type of coagulation factors, deficiency factor VIII, IX, and XI occur in hemophilic patients. Deficiency of those factors contributed to a certain type of hemophilia, namely hemophilia A for factor VIII deficiency, hemophilia B for factor IX deficiency, and hemophilia C for factor XI deficiency. ${ }^{2}$ From 172.373 hemophilic cases worldwide in 2012, about $80 \%$ cases were hemophilia A, 16\% cases were hemophilia $B$, and the rest was hemophilia $C$ and other bleeding disorders. Because it is an x-linked disease, hemophilia is more common found in male than female. Moreover, incidence of this disease in children is quite high, about 1 in 5.000 children. $^{3}$

Furthermore, clinical manifestation in hemophilia is bleeding. Bleeding can occur spontaneously or is caused by any injury or surgery. Bleeding may happen in many parts of the body, usually it is found in gum, nose (epistaxis), skin (echymosis), and joints either in upper or lower extremities which may cause hematoma etc. Bleeding can also occur in vital organs such as kidney (causing hematuria), gastrointestinal tract (causing hematemesis), and the most dangerous bleeding is in the retroperitoneal space, causing blood accumulation in abdomen.,6

Correspondence: Dina Marlina, Faculty of Medicine, Universitas Padjadjaran, Jalan Raya Bandung-Sumedang Km.21, Jatinangor, Sumedang, Indonesia, Phone: +6287821281609 Email: dinamarlinaa@gmail.com 
Besides the bleeding sign, the patient will experience pain in joint, causing immobility. Additionally, laboratory abnormality also found such as factor level, sometimes hemoglobin, hematocrit level, and platelet deficiency, and also prolonged Activated Partial Thromboplastin Time (APTT). ${ }^{4}$

Currently, management for hemophilia is only limited by replacement therapy and symptomatic drugs, which means that there is no definitive therapy yet. So, aggravating sign and symptoms have to be prevented starting from the first time the patients come. One of the ways to provide better care is by knowing the characteristics of each clinical manifestation and the likelihood of severity. The aim of this study was to identify the severity likelihood of clinical manifestation in hemophilic patients.

\section{Methods}

A retrospective cross-sectional study was carried out during August-October 2014 at the Hemato-Oncology Child Health Department, Dr. Hasan Sadikin General Hospital Bandung. A total of 87 medical records of hospitalized hemophilic patients from 2009 to 2013 were succesfully collected, but only $43(49.42 \%)$ medical records met the inclusion criteria, which were a complete patient identity (name, age, sex), written chief complaint, complete physical examination (bleeding, edema, hematoma, hemarthrosis, anemic symptoms) and laboratory test result (factor level, hemoglobin, hematocrit, platelet and APTT). Forty four samples (50.57\%) were excluded due to incomplete data and having other chronic diseases affecting the laboratory result such as typhoid, dengue, meningitis, thalassemia, TBC, etc. ${ }^{7}$

The data was collected from AugustOctober 2014 and was classified into hemophilia A, B, and C. Next, characteristics of clinical manifestation based on the severity were distributed to each type of hemophilia. The result was a frequency distribution of each type of hemophilia with severity likelihood for every clinical manifestation and laboratory findings. Meanwhile, Ethical clearance has been approved by the Health Research Ethics Committee, Faculty of Medicine, Universitas Padjadjaran in order to access the patient medical records.

\section{Result}

Most of the hospitalized hemophilic patients
Table 1 Characteristics and Type of Hospitalized Hemophilic Patients

\begin{tabular}{lc}
\hline $\begin{array}{c}\text { Characteristic and Type } \\
\text { of Hemophilia }\end{array}$ & $\begin{array}{c}\text { Total (\%) } \\
\text { (n=43) }\end{array}$ \\
\hline Age (years) & \\
$0-4$ & $14(33)$ \\
$5-10$ & $25(58)$ \\
$11-14$ & $4(9)$ \\
Sex & \\
Male & $42(98)$ \\
Female & $1(2)$ \\
Type of Hemophilia & \\
Hemophilia A & $35(81.4)$ \\
Hemophilia B & $4(9.3)$ \\
Hemophilia C & $4(9.3)$ \\
\hline
\end{tabular}

who met the inclusion criteria were 5-10 years old and male. In terms of the type of hemophilia, most of the patients had hemophilia A (Table 1).

As mentioned in the introduction, the most common complaint of hemophilia is bleeding. This study showed that about $58.14 \%$ cases had external bleeding, followed by edema. Hematoma and joint pain only occurred in a small percentage (Table 2).

The factor level deficiency proved to be the main cause for hemophilic patients to experience bleeding. Among 43 patients, 38 $(88 \%)$ cases were classified as severe factor deficiency, In terms of bleeding severity, 19 $(44 \%)$ cases were distributed in moderate level, the remaining (mild and severe level) was almost equally distributed. The hemophilic patients are commonly tested for anemic sign due to blood lost. The anemic state is seen from the result of the measurement of hemoglobin

Table 2 Chief Complaint of Hospitalized Hemophilic Patients ( $n=43)$

\begin{tabular}{lc}
\hline \multicolumn{1}{c}{ Chief complaint } & Total (\%) \\
\hline Bleeding (external) & $25(58.14)$ \\
Edema & $12(27.90)$ \\
Hematoma & $4(9.30)$ \\
Joint pain & $1(2.33)$ \\
Unconsciousness & $1(2.33)$ \\
Total & $43(100 \%)$ \\
\hline
\end{tabular}


Table 3 Clinical Manifestation and Laboratory Findings based on Type of Hemophilia

\begin{tabular}{|c|c|c|c|c|c|}
\hline \multirow{2}{*}{$\begin{array}{c}\text { Clinical } \\
\text { Manifestation }\end{array}$} & \multirow{2}{*}{ Severity } & \multicolumn{3}{|c|}{ Hemophilia } & \multirow{2}{*}{ Total } \\
\hline & & $A(\%)$ & $\mathrm{B}(\%)$ & $\mathrm{C}(\%)$ & \\
\hline \multirow[t]{3}{*}{ Factor Level } & Mild (6-46\%) & $1(2)$ & - & - & $1(2)$ \\
\hline & Moderate (1-5\%) & $3(7)$ & $1(2)$ & - & $4(9)$ \\
\hline & Severe $(<1 \%)$ & $31(72)$ & $3(7)$ & $4(9)$ & $38(88)$ \\
\hline \multirow[t]{3}{*}{ Bleeding } & Mild (no spontaneous bleeding) & $11(26)$ & $1(2)$ & - & $12(28)$ \\
\hline & $\begin{array}{l}\text { Moderate (sometimes spontaneous } \\
\text { bleeding) }\end{array}$ & $13(30)$ & $3(7)$ & $3(7)$ & $19(44)$ \\
\hline & Severe (often spontaneous bleeding) & $11(26)$ & - & $1(2)$ & $12(28)$ \\
\hline \multirow[t]{4}{*}{ Hemoglobin level } & Normal ( $\geq 115$ g/l) & $15(35)$ & $1(2)$ & $2(5)$ & $18(42)$ \\
\hline & Mild (110-114 g/l) & $2(5)$ & $1(2)$ & - & $3(7)$ \\
\hline & Moderate (80-109 g/l) & $4(9)$ & $2(5)$ & $1(2)$ & 7 (16) \\
\hline & Severe $(\leq 80 \mathrm{~g} / \mathrm{l})$ & $14(33)$ & - & $1(2)$ & $15(35)$ \\
\hline \multirow[t]{4}{*}{ Hematocrit level } & Normal (35-48\%) & $10(23)$ & $1(2)$ & $2(5)$ & $13(30)$ \\
\hline & Mild (32-35\%) & $9(21)$ & - & - & $9(21)$ \\
\hline & Moderate (28-32\%) & $2(5)$ & $2(5)$ & - & $4(7)$ \\
\hline & Severe $(<28 \%)$ & $14(33)$ & $1(2)$ & $2(5)$ & $17(40)$ \\
\hline \multirow[t]{2}{*}{ Platelet level } & Normal (150.000-450.000/mm3) & $25(58)$ & - & $3(7)$ & $28(65)$ \\
\hline & Increase $(>450.000 / \mathrm{mm} 3)$ & $10(23)$ & $4(9)$ & $1(2)$ & $15(34)$ \\
\hline \multirow[t]{3}{*}{ APTT } & Normal (18-28 second) & $1(2)$ & - & $1(2)$ & $2(4)$ \\
\hline & Mild (<40 second) & $1(2)$ & $1(2)$ & - & $2(4)$ \\
\hline & Moderate to severe (>40 second) & $33(77)$ & $3(7)$ & $3(7)$ & $39(91)$ \\
\hline
\end{tabular}

and hematocrit level. Most of the cases had mild to severe anemia and only $42 \%$ cases had no anemia. This situation also occurred in hematocrit level. Moreover, the platelet count in most of the cases was in normal value. Moreover, an interesting result was discovered in this study, about $91 \%$ cases had prolonged APTT in moderate to severe level.

\section{Discussion}

This study showed that most of the hospitalized hemophilic patients in Dr Hasan Sadikin General Hospital from 2009-2013 had hemophilia A. This result was similar with previous studies conducted by the World Federation for Hemophilia (WFH) ${ }^{3}$ and Josephson ${ }^{8}$ which stated that among the total of hemophilia patients, more than $80 \%$ of them were hemophilia A. A study by Shawky et al. ${ }^{9}$ recorded that there were more Hemophilia A patients (6.26\%) than hemophilia B patients $(0.55 \%)$ at Pediatric clinic, Ain-Shams University hospitalin in Egypt.

The most common clinical sign that become the chief complaint of the patients was external bleeding. This finding is similar with a study conducted by Liu et al. ${ }^{7}$

Besides this study discovered the severity level of various laboratory test results. Most of the cases had severe factor deficiency. Similar result was discovered in a study carried out by the National Hemophilia Foundation (NHF) ${ }^{10}$. About $60-70 \%$ from the total hemophilic population worldwide is in the severe factor level deficiency. Regarding the type of hemophilia, a previous study by Liras et al. $^{2}$ showed that $40 \%$ of cases of hemophilia A and hemophilia B, respectively, contributes to severe level of factor deficiency. In this study, about $75-80 \%$ of patients for each type was distributed in the severe level. On the other hand, another study conducted by Ghaniema et al. ${ }^{11}$ showed a different result. They mentioned that $60 \%$ of their samples were in the moderate level of factor deficiency.

Moreover, the bleeding sign was also classified into mild, moderate and severe level. The bleeding sign for hemophilic patients who were hospitalized in Dr. Hasan Sadikin General 
Hospital from 2009 to 2013 was distributed in moderate level. This study showed that most of the Hemophilia A cases had moderate to severe level of bleeding compared to the Hemophilia B cases that had mild to moderate level of bleeding severity. Additionally, Fasulo et al. ${ }^{12}$ stated that hemophilia B had a milder manifestation than hemophilia A.

Another laboratory test for hemophilia patients was the measurement of hemoglobin level. This study discovered that most of the patients had mild to severe level of anemia, although there were still $42 \%$ cases that had no anemia. Besides, significant hemoglobin and hematocrit level decrement resulted in anemic sign and were related to bleeding sign. A previous study conducted by Buchanan and Holtkamp ${ }^{13}$ stated that hemoglobin level decrement could occur in hemophilic patients, while in fact, blood lost due to bleeding in hemophilia rarely causes anemia. There was another factor causing anemia in hemophilia cases which was still unknown.

The number of patients with a normal platelet level was higher than a decreased or increased platelet level. The interesting finding discovered in this study was that the platelet level mostly increased. A study conducted by $\mathrm{Yee}^{14}$ stated that the platelet level decrement likelihood would be found in severe hemophilia unless hemophilia is still in a mild stage.

Furthermore, APPT prolongation occurred in most cases. This condition occurred due to the severity of factor level deficiency or bleeding. If the factor level deficiency and bleeding sign were in severe level, it would cause significant APTT prolongation. Previous studies conducted by Tantawy ${ }^{6}$ and Cuker et al. ${ }^{15}$ stated that APTT would be normal in mild hemophilia while prolonged APTT would be found in severe hemophilia.

This study still had limitations. The sample size was very small, so it was difficult to compare the severity of each type of hemophilia. Moreover, this study was carried out in a top referral hospital so it was suspected that most cases which came to this hospital were already in the moderate to severe state.

It can be concluded that most of the hospitalized hemophilic patients has hemophilia A. Most of the patients has moderate to severe bleeding with laboratory test result between moderate to severe level as well.

\section{References}

1. Hussain R, Abid NB, Hussain S, Shaukat Z, Altaf M, Altaf S, et al. Three novel F8 mutations in sporadic haemophilia A cases. SpringerPlus. 2012;1:10.

2. Liras A, Segovia C, Gabán AS. Advanced therapies for the treatment of hemophilia: future perspectives. Orphanet J Rare Dis. 2012;7:97.

3. World Federation of Hemophilia. Report on the Annual Global Survey 2012. Montrel, Quebec: World Federation of Hemophilia; 2013.

4. Davidson CS, Epstein RD, Miller GF, Taylor FHL. Hemophilia, a clinical study of 40 patients. Blood. 1949;4(2):97-119.

5. Jayandharan G, Srivastava A. Hemophilia: disease, diagnosis and treatment. J Genet Syndr Gene Ther S. 2011;S1:005.

6. Tantawy AA. Molecular genetics of hemophilia A: clinical perspectives. Egyptian Journal of Medical Human Genetics. 2010;11(2):105-14.

7. Liu B, Liu L, Feng Y, Li L. A case report on the surgical treatment of the huge inflammatory pseudotumor in the AIDS patient with hemophilic. Case Rep Pathol. 2011;2011:798649.

8. Josephson N. The hemophilias and their clinical management. Hematology Am Soc Hematol Educ Program. 2013;2013:261-7.

9. Shawky R M, Elsayed NS, Ibrahim DS, Seifeldin NS.. Profile of genetic disorders prevalent in northeast region of Cairo, Egypt. Egyptian Journal of Medical Human Genetics. 2012;13(1):45-62.

10. National Hemophilia Foundation. Hemophilia A (Factor VIII Deficiency) [cited 2014 February 15]. Available from: http://www.hemophilia.org/NHFWeb/ MainPgs/MainNHF.aspx?menuid=180\&co ntentid $=45 \&$ rptname=bleeding.

11. Ghaniema ME, Ahmed FS, Amin RI, Ayoub SM. Evaluation of osteoporosis in hemophilic arthropathy patients: correlation with disease severity and serum trace minerals. J Osteoporos. 2011;2011:106380.

12. Fasulo MR, Mancuso ME, Chantarangkul $\mathrm{V}$, Cannavò $\mathrm{A}$, Clerici $\mathrm{M}$, Padovan $\mathrm{L}$, et al. Is hemophilia B less severe than hemophilia A? results of global coagulation assays. Blood. 2013;122(21):2352.

13. Buchanan GR, Holtkamp CA. Reduced hemoglobin values in children and young adults with hemophil. Pediatrics.1988;81(6):840-5

14. Yee DL. Platelets as modifiers of clinical phenotype in hemophilia. 
Dina Marlina, Lelani Reniarti, Fifi Veronica: Five-Year Data of Clinical Characteristics and Laboratory Findings 609 of Hospitalized Hemophilic Patients in Dr. Hasan Sadikin General Hospital

TheScientificWorldJOURNAL. 2006;6:6618.

15. Cuker A, Connors JM, Katz JT, Levy BD,
Loscalzo J. Clinical problem solving: a bloody mystery. N Engl J Med. 2009;361:1887-94. 